\title{
A Linear Homogeneous Partial Differential Equation with Entire Solutions Represented by Laguerre Polynomials
}

\author{
Xin-Li Wang, ${ }^{1}$ Feng-Li Zhang, ${ }^{1}$ and Pei-Chu $\mathrm{Hu}^{2}$ \\ ${ }^{1}$ College of Science, University of Shanghai for Science and Technology, Shanghai 200093, China \\ ${ }^{2}$ Department of Mathematics, Shandong University, Shandong, Jinan 250100, China
}

Correspondence should be addressed to Xin-Li Wang, xlwang602@163.com

Received 11 November 2011; Revised 19 March 2012; Accepted 19 March 2012

Academic Editor: Agacik Zafer

Copyright (C) 2012 Xin-Li Wang et al. This is an open access article distributed under the Creative Commons Attribution License, which permits unrestricted use, distribution, and reproduction in any medium, provided the original work is properly cited.

We study a homogeneous partial differential equation and get its entire solutions represented in convergent series of Laguerre polynomials. Moreover, the formulae of the order and type of the solutions are established.

\section{Introduction and Main Results}

The existence and behavior of global meromorphic solutions of homogeneous linear partial differential equations of the second order

$$
a_{0} \frac{\partial^{2} u}{\partial t^{2}}+2 a_{1} \frac{\partial^{2} u}{\partial t \partial z}+a_{2} \frac{\partial^{2} u}{\partial z^{2}}+a_{3} \frac{\partial u}{\partial t}+a_{4} \frac{\partial u}{\partial z}+a_{6} u=0
$$

where $a_{k}=a_{k}(t, z)$ are polynomials for $(t, z) \in \mathbb{C}^{2}$, have been studied by Hu and Yang [1]. Specially, in $[1,2]$, they have studied the following cases of (1.1)

$$
\begin{gathered}
t^{2} \frac{\partial^{2} u}{\partial t^{2}}-z^{2} \frac{\partial^{2} u}{\partial z^{2}}+(2 t+2) \frac{\partial u}{\partial t}-2 z \frac{\partial u}{\partial z}=0 \\
t^{2} \frac{\partial^{2} u}{\partial t^{2}}-z^{2} \frac{\partial^{2} u}{\partial z^{2}}+t \frac{\partial u}{\partial t}-z \frac{\partial u}{\partial z}+t^{2} u=0
\end{gathered}
$$


and showed that the solutions of (1.2) and (1.3) are closely related to Bessel functions and Bessel polynomials, respectively. $\mathrm{Hu}$ and $\mathrm{Li}$ [3] studied meromorphic solutions of homogeneous linear partial differential equations of the second order in two independent complex variables:

$$
\left(1-t^{2}\right) \frac{\partial^{2} u}{\partial t^{2}}+z^{2} \frac{\partial^{2} u}{\partial z^{2}}-\{\alpha-\beta+(\alpha+\beta+2) t\} \frac{\partial u}{\partial t}+(\alpha+\beta+2) z \frac{\partial u}{\partial z}=0
$$

where $\alpha, \beta \in \mathbb{C}$. Equation (1.4) has a lot of entire solutions on $\mathbb{C}^{2}$ represented by Jacobian polynomials. Global solutions of some first-order partial differential equations (or system) were studied by Berenstein and Li [4], Hu and Yang [5], Hu and Li [6], Li [7], Li and Saleeby [8], and so on.

In this paper, we concentrate on the following partial differential equation (PDE)

$$
t \frac{\partial^{2} u}{\partial t^{2}}+(\alpha+1-t) \frac{\partial u}{\partial t}+z \frac{\partial u}{\partial z}=0
$$

for a real $\alpha>0$. We will characterize the entire solutions of (1.5), which are related to Laguerre polynomials. Further, the formulae of the order and type of the solutions are obtained.

It is well known that the Laguerre polynomials are defined by

$$
L_{n}(\alpha, t)=\sum_{k=0}^{n}\left(\begin{array}{l}
n+\alpha \\
n-k
\end{array}\right) \frac{(-t)^{k}}{k !}
$$

which are solutions of the following ordinary differential equations (ODE):

$$
t \frac{d^{2} \omega}{d t^{2}}+(\alpha+1-t) \frac{d \omega}{d t}+n \omega=0
$$

Moreover, $\mathrm{Hu}$ [9] pointed out that the generating function of $L_{n}(\alpha, t)$

$$
F(\alpha, t, z)=(1-z)^{-\alpha-1} e^{-t z /(1-z)}=\sum_{n=0}^{\infty} L_{n}(\alpha, t) z^{n}
$$

is a solution of the PDE (1.5). Based on the methods from Hu and Yang [2], we get the following results.

Theorem 1.1. The partial differential equation (1.5) has an entire solution $u=f(t, z)$ on $\mathbb{C}^{2}$, if and only if $u=f(t, z)$ has a series expansion

$$
f(t, z)=\sum_{n=0}^{\infty} c_{n} L_{n}(\alpha, t) z^{n}
$$


such that

$$
\limsup _{n \rightarrow \infty} \sqrt[n]{\left|c_{n}\right|}=0
$$

If $f(t, z)$ is an entire function on $\mathbb{C}^{2}$, set

$$
M(r, f)=\max _{|t| \leq r,|z| \leq r}|f(t, z)|
$$

we define its order by

$$
\operatorname{ord}(f)=\limsup _{r \rightarrow \infty} \frac{\log ^{+} \log ^{+} M(r, f)}{\log r}
$$

where

$$
\log ^{+} x= \begin{cases}\log x, & \text { if } x \geq 1 \\ 0, & \text { if } x<1\end{cases}
$$

Theorem 1.2. If $f(t, z)$ is defined by (1.9) and (1.10), then

$$
\rho \leq \operatorname{ord}(f) \leq \max (1, \rho)
$$

where

$$
\rho=\limsup _{n \rightarrow \infty} \frac{\log n}{\log \left(1 / \sqrt[n]{\left|c_{n}\right|}\right)}
$$

Valiron [10] showed that each entire solution of a homogeneous linear ODE with polynomial coefficients was of finite order. By studying (1.2) and (1.3), Hu and Yang showed that Valiron's theorem was not true for general partial differential equations. Here by using Theorems 1.1 and 1.2, we can construct entire solution of (1.5) with arbitrary order $\rho(\rho \geq 1)$.

If $0<\lambda=\operatorname{ord}(f)<\infty$, we define the type of $f$ by

$$
\operatorname{typ}(f)=\limsup _{r \rightarrow \infty} \frac{\log ^{+} M(r, f)}{r^{\curlywedge}}
$$

Theorem 1.3. If $f(t, z)$ is defined by (1.9) and (1.10), and $1<\lambda=\operatorname{ord}(f)<\infty$, then the type $\sigma=\operatorname{typ}(f)$ satisfies

$$
e \sigma \lambda=\limsup _{n \rightarrow \infty} n \sqrt[n]{\left|c_{n}\right|^{\lambda}}
$$


Lindelöf-Pringsheim theorem [11] gave the expression of order and type for one complex variable entire function, and for two variable entire function the formulae of order and type were obtained by Bose and Sharma in [12]. Hu and Yang [2] established an analogue of Lindelöf-Pringsheim theorem for the entire solution of PDE (1.2). But from Theorems 1.2 and 1.3, we find that the analogue theorem for the entire solution of (1.5) is different from the results due to $\mathrm{Hu}$ and Yang.

\section{An Estimate of Laguerre Polynomials}

Before we prove our theorems, we give an upper bound of $L_{n}(\alpha, t)$, which will play an important role in this paper. The following asymptotic properties of $L_{n}(\alpha, t)$ can be found in [13]:

(a)

$$
L_{n}(\alpha, t)=\frac{1}{2 \sqrt{\pi}} e^{t / 2}(-t)^{-\alpha / 2-1 / 4} n^{\alpha / 2-1 / 4} e^{2 \sqrt{-n t}}\left(1+O\left(n^{-1 / 2}\right)\right) \quad(n \longrightarrow \infty)
$$

holds for $t$ in the complex plane cut along the positive real semiaxis; thus, for $|t| \leq r$, we obtain that

$$
\left|L_{n}(\alpha, t)\right| \leq n^{\alpha / 2-1 / 4} e^{r / 2} r^{-\alpha / 2-1 / 4} e^{2 \sqrt{n r}}
$$

holds when $n$ is large enough.

(b)

$$
\frac{L_{n}(\alpha, t)}{n^{\alpha / 2}}=e^{t / 2} t^{-\alpha / 2} J_{\alpha}(2 \sqrt{n t})+O\left(n^{-3 / 4}\right) \quad(n \longrightarrow \infty)
$$

holds uniformly on compact subsets of $(0,+\infty)$, where $J_{\alpha}$ is the Bessel function and

$$
J_{\alpha}(2 \sqrt{n t})=\frac{2}{\sqrt{\pi} \Gamma(\alpha+1 / 2)}(\sqrt{n t})^{\alpha} \int_{0}^{\pi / 2} \cos (\sqrt{n t} \cos x) \sin ^{2 \alpha} x \mathrm{~d} x
$$

combining with (2.3), for $|t| \leq r$ we can deduce that

$$
\left|L_{n}(\alpha, t)\right| \leq \frac{\pi n^{\alpha}}{\sqrt{\pi} \Gamma(\alpha+1 / 2)} e^{t / 2} \leq \frac{\pi n^{\alpha}}{\sqrt{\pi} \Gamma(\alpha+1 / 2)} e^{r / 2} \leq n^{\alpha / 2-1 / 4} e^{r / 2} r^{-\alpha / 2-1 / 4} e^{2 \sqrt{n r}}
$$

holds when $n$ is large enough. Then (2.2) and (2.5) imply

$$
M\left(r, L_{n}\right) \leq n^{\alpha / 2-1 / 4} e^{r / 2} r^{-\alpha / 2-1 / 4} e^{2 \sqrt{n r}},
$$

where

$$
M\left(r, L_{n}\right)=\max _{|t| \leq r}\left|L_{n}(\alpha, t)\right| .
$$




\section{Proof of Theorem 1.1}

Assuming that $u=f(t, z)$ is an entire solution on $\mathbb{C}^{2}$ satisfying (1.5), we have Taylor expansion

$$
f(t, z)=\sum_{n=0}^{\infty} \frac{w_{n}(t)}{n !} z^{n}
$$

where

$$
w_{n}(t)=\frac{\partial^{n} f}{\partial z^{n}}(t, 0)
$$

Hence $w_{n}(t)$ is an entire solution of (1.7).

By the method of Frobenius (see [14]), we can get a second independent solution $X_{n}(\alpha, t)$ of (1.7) which is

$$
X_{n}(\alpha, t)=q L_{n}(\alpha, t) \log t+\sum_{i=0}^{\infty} p_{i} t^{i}
$$

where $q(\neq 0), p_{i}$ are constants.

So there exist $c_{n}$ and $b_{n}$ satisfying

$$
w_{n}(t)=n ! c_{n} L_{n}(\alpha, t)+b_{n} X_{n}(\alpha, t) .
$$

Because of the singularity of $X_{n}(\alpha, t)$ at $t=0$, we obtain $b_{n}=0$. That shows

$$
f(t, z)=\sum_{n=0}^{\infty} c_{n} L_{n}(\alpha, t) z^{n}
$$

Now we need to estimate the terms of $c_{n}$. Since

$$
f(0, z)=\sum_{n=0}^{\infty} c_{n} L_{n}(\alpha, 0) z^{n}
$$

is an entire function, we have

$$
\limsup _{n \rightarrow \infty} \sqrt[n]{\left|c_{n}\right| L_{n}(\alpha, 0)}=0 .
$$

Since

$$
L_{n}(\alpha, 0)=\left(\begin{array}{c}
n+\alpha \\
n
\end{array}\right) \approx \frac{n^{\alpha}}{\Gamma(\alpha+1)}
$$

we easily get

$$
\limsup _{n \rightarrow \infty} \sqrt[n]{\left|c_{n}\right|}=0
$$


Conversely, the relations (1.7), (1.9), and (1.10) imply that

$$
t \frac{\partial^{2} f}{\partial t^{2}}+(\alpha+1-t) \frac{\partial f}{\partial t}+z \frac{\partial f}{\partial z}=\sum_{n=0}^{\infty} c_{n}\left\{t \frac{d^{2} L_{n}(\alpha, t)}{d t^{2}}+(\alpha+1-t) \frac{d L_{n}(\alpha, t)}{d t}+n L_{n}(\alpha, t)\right\} z^{n}=0
$$

holds for all $(t, z) \in \mathbb{C}^{2}$. Since (2.6) implies

$$
\limsup _{n \rightarrow \infty} \sqrt[n]{M\left(r, L_{n}\right)} \leq 1
$$

we have

$$
\limsup _{n \rightarrow \infty} \sqrt[n]{\left|c_{n} L_{n}(\alpha, t)\right|} \leq \limsup _{n \rightarrow \infty} \sqrt[n]{\left|c_{n}\right| M\left(r, L_{n}\right)} \leq \limsup _{n \rightarrow \infty} \sqrt[n]{\left|c_{n}\right|}
$$

Combining (1.10), (3.10) with (3.12), we can get that $u=f(t, z)$ is obviously an entire solution of (1.5) on $\mathbb{C}^{2}$.

\section{Proof of Theorem 1.2}

Firstly, we prove $\rho \leq \operatorname{ord}(f)$. If $\rho=0$, the result is trivial. Now we assume $0<\rho \leq \infty$ and prove ord $(f) \geq k_{1}$ for any $0<k_{1}<\rho$. The relation (1.15) implies that there exists a sequence $n_{j} \rightarrow \infty$ such that

$$
n_{j} \log n_{j} \geq k_{1} \log \frac{1}{\left|c_{n_{j}}\right|}
$$

By using Cauchy's inequality of holomorphic functions, we have

$$
\left|\frac{\partial^{n} f}{\partial z^{n}}(0,0)\right| \leq n ! r^{-n} M(r, f)
$$

together with the formula of the coefficients of the Taylor expansion

$$
\frac{\partial^{n} f}{\partial z^{n}}(0,0)=c_{n} L_{n}(\alpha, 0) n !
$$

we obtain $M(r, f) \geq\left|c_{n} L_{n}(\alpha, 0)\right| r^{n}$. Since $\left|L_{n}(\alpha, 0)\right| \geq 1$, we have

$$
\left|c_{n}\right| r^{n} \leq M(r, f)
$$


then

$$
\log M(r, f) \geq \log \left|c_{n}\right|+n \log r \geq n_{j}\left(\log r-\frac{1}{k_{1}} \log n_{j}\right)
$$

Putting $r_{j}=\left(e n_{j}\right)^{1 / k_{1}}$, we have

$$
\log M\left(r_{j}, f\right) \geq \frac{r_{j}^{k_{1}}}{e k_{1}}
$$

which means ord $(f) \geq k_{1}$. Then we can get $\operatorname{ord}(f) \geq \rho$.

Next, we will prove ord $(f) \leq \max (1, \rho)$. Set $\rho^{\prime}=\max (1, \rho)$. The result is easy for $\rho^{\prime}=\infty$; then we assume $\rho^{\prime}<\infty$. For any $\varepsilon>0$, (1.15) implies that there exists $n_{0}>0$, when $n>n_{0}$, we have

$$
\left|c_{n}\right|<n^{-n(1+2 \varepsilon) / \rho^{\prime}(1+3 \varepsilon)}=n^{-n(1+2 \varepsilon) / k_{2}},
$$

where $k_{2}=\rho^{\prime}(1+3 \varepsilon)>1$. For any $\alpha \geq 0$, there exists $n_{1}>n_{0}$ such that when $n>n_{1}$,

$$
n^{(\alpha / 2-1 / 4)}<n^{n\left(\varepsilon / k_{2}\right)},
$$

combining with (2.6) and (4.7), we get

$$
\begin{aligned}
M(r, f) & \leq \sum_{n=0}^{\infty}\left|c_{n}\right| M\left(r, L_{n}\right) r^{n} \\
& \leq C r^{2 n_{1}}+C \sum_{n>n_{1}}^{\infty}\left|c_{n}\right| n^{\alpha / 2-1 / 4} e^{r / 2} e^{2 \sqrt{n r}} r^{n} \\
& \leq C r^{2 n_{1}}+C e^{r / 2} \sum_{n>n_{1}}^{\infty} n^{-(1+\varepsilon) n / k_{2}} e^{2 \sqrt{n r}} r^{n}
\end{aligned}
$$

where $C$ is a constant but not necessary to be the same every time.

Set $m_{1}(r)=((1 / \varepsilon)(2 \sqrt{r} / \log r))^{2}$, which means that $e^{2 \sqrt{n r}}<r^{\varepsilon n}$ for $n>m_{1}(r)$. Further set $m_{2}(r)=(2 r)^{k_{2}}$, which yields that when $n \geq m_{2}(r)$,

$$
\left(n^{-1 / k_{2}} r\right)^{(1+\varepsilon) n} \leq\left(\frac{1}{2}\right)^{(1+\varepsilon) n}<\left(\frac{1}{2}\right)^{n}
$$

Obviously, we can choose $r_{0}>0$ such that $m_{2}(r)>m_{1}(r)$ for $r>r_{0}$. Then

$$
\sum_{n>m_{2}(r)} n^{-(1+\varepsilon) n / k_{2}} e^{2 \sqrt{n r}} r^{n} \leq \sum_{n>m_{2}(r)}^{\infty} n^{-(1+\varepsilon) n / k_{2}} r^{(1+\varepsilon) n} \leq \sum_{n>m_{2}(r)}^{\infty}\left(\frac{1}{2}\right)^{n} \leq 1 .
$$


We also have

$$
\begin{aligned}
\sum_{n_{1}<n \leq m_{2}(r)} n^{-(1+\varepsilon) n / k_{2}} e^{2 \sqrt{n r}} r^{n} & \leq \sum_{n_{1}<n \leq m_{2}(r)} n^{-(1+\varepsilon) n / k_{2}} e^{2 \sqrt{m_{2}(r) r}} r^{m_{2}(r)} \\
& \leq \sum_{n_{1}<n \leq m_{2}(r)} n^{-(1+\varepsilon) n / k_{2}} r^{m_{2}(r)} r^{\varepsilon m_{2}(r)} \\
& \leq r^{(1+\varepsilon) m_{2}(r)} \sum_{n_{1}<n \leq m_{2}(r)} n^{-(1+\varepsilon) n / k_{2}} \\
& \leq C r^{(1+\varepsilon) m_{2}(r)} \\
& =C r^{(1+\varepsilon)(2 r)^{k_{2}}} .
\end{aligned}
$$

Therefore, when $|t|=r \geq r_{0}$, we have

$$
M(r, f) \leq C r^{2 n_{1}}+C e^{r / 2}+C e^{r / 2} r^{(1+\varepsilon)(2 r)^{k_{2}}},
$$

which means ord $(f) \leq k_{2}$. Hence ord $(f) \leq \rho^{\prime}=\max (1, \rho)$ follows by letting $\varepsilon \rightarrow 0$.

\section{Proof of Theorem 1.3}

Set

$$
\kappa=\limsup _{n \rightarrow \infty} n \sqrt[n]{\left|c_{n}\right|^{\lambda}}
$$

At first, we prove $e \lambda \sigma \geq \kappa$. The result is trivial for $\kappa=0$, we assume $0<\kappa \leq \infty$ and take $\varepsilon$ with $0<\varepsilon<\kappa$, set

$$
k_{3}= \begin{cases}\kappa-\varepsilon, & \text { if } \kappa<\infty \\ \frac{1}{\varepsilon}, & \text { if } \kappa=\infty\end{cases}
$$

Equation (5.1) implies that there exists a sequence $n_{j} \rightarrow \infty$ satisfying

$$
\left|c_{n_{j}}\right|>\left(\frac{k_{3}}{n_{j}}\right)^{n_{j} / \lambda}
$$

combining with (4.4), we can deduce that

$$
M(r, f) \geq\left|c_{n_{j}}\right| r^{n_{j}} \geq\left(\frac{k_{3}}{n_{j}}\right)^{n_{j} / \lambda} r^{n_{j}}=\left(\frac{k_{3}}{n_{j}} r^{\lambda}\right)^{n_{j} / \lambda} .
$$

Taking $r_{j}^{\curlywedge}=e n_{j} / k_{3}$, we get $M\left(r_{j}, f\right)>e^{k_{3} r_{j}^{\curlywedge} / e \curlywedge}$, which yields $\sigma \geq k_{3} / e \lambda$, so $e \lambda \sigma \geq \kappa$. 
Next, we prove $e \lambda \sigma \leq \kappa$. We may assume $\kappa<\infty$. Equation (5.1) implies that for any $\varepsilon>0$, there exists $n_{0}>0$, such that when $n>n_{0}$,

$$
\left|c_{n}\right|<\left(\frac{\kappa+(\varepsilon / 2)}{n}\right)^{n / \mathcal{A}}
$$

For any $\alpha \geq 0$, we choose $n_{1}\left(>n_{0}\right)$ such that when $n>n_{1}$,

$$
n^{\alpha / 2-1 / 4}<\left(\frac{\kappa+\varepsilon}{\kappa+(\varepsilon / 2)}\right)^{n / \lambda}
$$

combining with (2.6), we have

$$
\begin{aligned}
M(r, f) \leq & \sum_{n=0}^{\infty}\left|c_{n}\right| M\left(r, L_{n}\right) r^{n} \\
& \leq C r^{2 n_{1}}+C \sum_{n>n_{1}}^{\infty}\left|c_{n}\right| n^{\alpha / 2-1 / 4} e^{r / 2} e^{2 \sqrt{n r}} r^{n} \\
& \leq C r^{2 n_{1}}+C e^{r / 2} \sum_{n>n_{1}}^{\infty}\left(\frac{\kappa+\varepsilon}{n}\right)^{n / \lambda} e^{2 \sqrt{n r}} r^{n}
\end{aligned}
$$

Set $m_{3}(r)=16 r \lambda^{2}$, when $n>m_{3}(r)$, we deduce $e^{2 \sqrt{n r}}<e^{n / 2 \lambda}$. Set $m_{4}(r)=2(\kappa+\varepsilon) r^{\lambda}$, it is obvious that $(\kappa+\varepsilon) r^{\lambda} / n<1 / 2$ for $n>m_{4}(r)$. Since $\lambda>1$, there exists $r_{1}$ such that when $r>r_{1}$,

$$
m_{4}(r)=2(\kappa+\varepsilon) r^{\curlywedge}>16 r \lambda^{2}=m_{3}(r)
$$

Then

$$
\sum_{n>m_{4}(r)}^{\infty}\left(\frac{\kappa+\varepsilon}{n}\right)^{n / \lambda} e^{2 \sqrt{n r}} r^{n}=\sum_{n>m_{4}(r)}^{\infty}\left(\frac{(\mathcal{\kappa}+\varepsilon) r^{\lambda}}{n}\right)^{n / \lambda} e^{2 \sqrt{n r}} \leq \sum_{n>m_{4}(r)}^{\infty}\left(\frac{e}{4}\right)^{n / 2 \lambda} \leq C .
$$

We note that for $a>0, b>0, \max _{x>0}(a / x)^{x / b}=e^{a / e b}$, then we have

$$
\left(\frac{\kappa+\varepsilon}{n}\right)^{n / \lambda} r^{n}=\left(\frac{(\kappa+\varepsilon) r^{\lambda}}{n}\right)^{n / \lambda} \leq e^{(\kappa+\varepsilon) r^{\lambda} / e \lambda}
$$

This shows

$$
\begin{aligned}
\sum_{n_{1}<n \leq m_{4}(r)}\left(\frac{\kappa+\varepsilon}{n}\right)^{n / \lambda} e^{2 \sqrt{n r}} r^{n} & \leq m_{4}(r) e^{2 \sqrt{m_{4}(r) r}} e^{(\kappa+\varepsilon) r^{\lambda} / e \lambda} \\
& \leq 2(\kappa+\varepsilon) r^{\lambda} e^{2 \sqrt{2(\kappa+\varepsilon) r^{\lambda+1}}} e^{(\kappa+\varepsilon) r^{\lambda} / e \lambda}
\end{aligned}
$$


Therefore when $|t|=r \geq r_{1}$,

$$
M(r, f) \leq C e^{r / 2}(\kappa+\varepsilon) r^{\lambda} e^{2 \sqrt{2(\kappa+\varepsilon) r^{\lambda+1}}} e^{(\kappa+\varepsilon) r^{\lambda} / e \lambda}+C r^{2 n_{1}}+C e^{r / 2}
$$

Together with $\lambda>1$ and the definition of type, we can get $\sigma \leq(\kappa+\varepsilon) / e \lambda$, which yields $e \lambda \sigma \leq \kappa$ by letting $\varepsilon \rightarrow 0$.

\section{Acknowledgments}

The authors sincerely thank the reviewers for their valuable suggestions and useful comments that have led to the present improved version of the original paper. The first author was partially supported by Natural Science Foundation of China (11001057), and the third author was partially supported by Natural Science Foundation of Shandong Province.

\section{References}

[1] P.-C. Hu and C.-C. Yang, "Global solutions of homogeneous linear partial differential equations of the second order," Michigan Mathematical Journal, vol. 58, no. 3, pp. 807-831, 2009.

[2] P.-C. Hu and C.-C. Yang, "A linear homogeneous partial differential equation with entire solutions represented by Bessel polynomials," Journal of Mathematical Analysis and Applications, vol. 368, no. 1, pp. 263-280, 2010.

[3] P.-C. Hu and B.-Q. Li, "Unicity of meromorphic solutions of partial differential equations," Journal of Mathematical Sciences, vol. 173, pp. 201-206, 2011.

[4] C. A. Berenstein and B. Q. Li, "On certain first-order partial differential equations in $\mathbb{C}^{n}$," in Harmonic Analysis, Signal Processing, and Complexity, vol. 238, pp. 29-36, Birkhäuser Boston, Boston, Mass, USA, 2005.

[5] P. C. Hu and C.-C. Yang, "Malmquist type theorem and factorization of meromorphic solutions of partial differential equations," Complex Variables, vol. 27, no. 3, pp. 269-285, 1995.

[6] P.-C. Hu and B. Q. Li, "On meromorphic solutions of nonlinear partial differential equations of first order," Journal of Mathematical Analysis and Applications, vol. 377, no. 2, pp. 881-888, 2011.

[7] B. Q. Li, "Entire solutions of certain partial differential equations and factorization of partial derivatives," Transactions of the American Mathematical Society, vol. 357, no. 8, pp. 3169-3177, 2005.

[8] B. Q. Li and E. G. Saleeby, "Entire solutions of first-order partial differential equations," Complex Variables, vol. 48, no. 8, pp. 657-661, 2003.

[9] P.-C. Hu, Introduction of Function of One Complex Variable, Science Press, Beijing, China, 2008.

[10] G. Valiron, Lectures on the General Theory of Integral Functions, Ëdouard Privat, Toulouse, France, 1923.

[11] Y.-Z. He and X.-Z. Xiao, Algebroid Functions and Ordinary Differential Equations, Science Press, Beijing, China, 1988.

[12] S. K. Bose and D. Sharma, "Integral functions of two complex variables," Compositio Mathematica, vol. 15, pp. 210-226, 1963.

[13] G. Szegó, Orthogonal Polynomials, vol. 23 of of American Mathematical Society Colloquium Publications, American Mathematical Society, Providence, RI, USA, 4th edition, 1975.

[14] Z.-X. Wang and D.-R. Guo, Introduction to Special Function, Peking University Press, Beijing, China, 2000. 


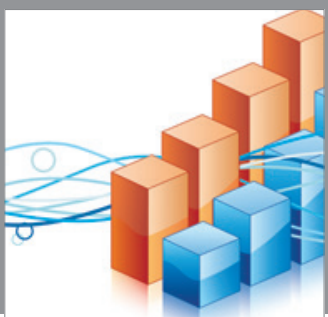

Advances in

Operations Research

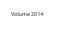

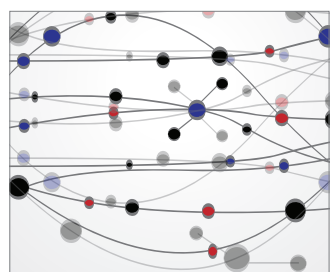

\section{The Scientific} World Journal
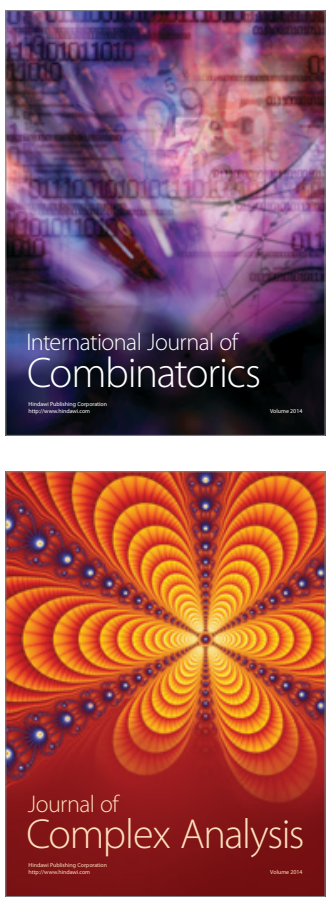

International Journal of

Mathematics and

Mathematical

Sciences
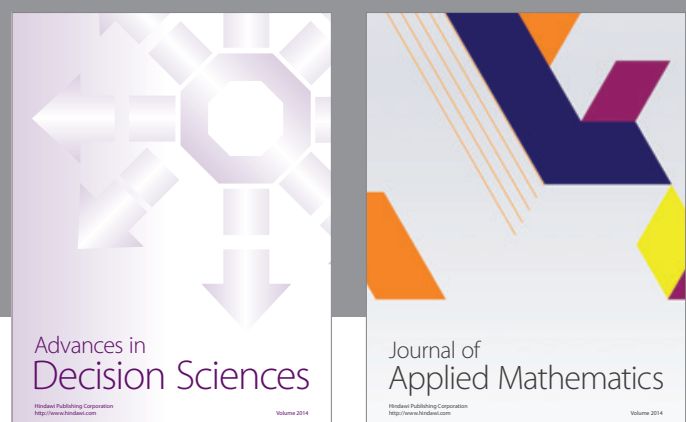

Journal of

Applied Mathematics
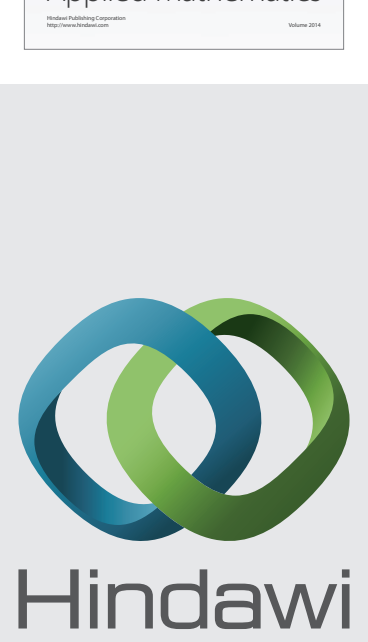

Submit your manuscripts at http://www.hindawi.com
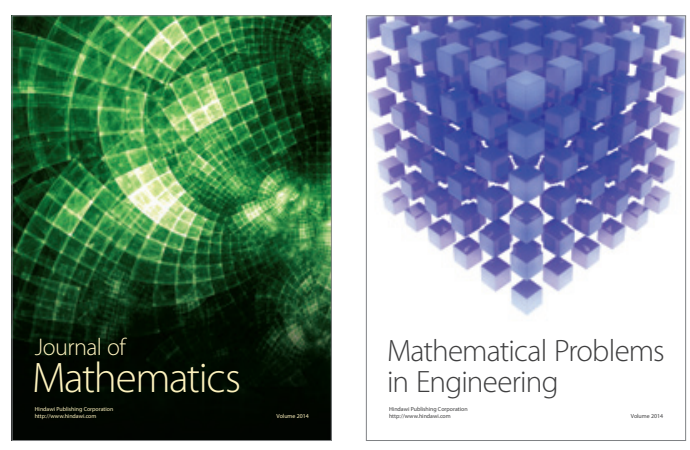

Mathematical Problems in Engineering
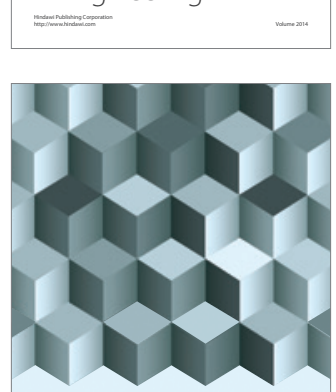

Journal of

Function Spaces
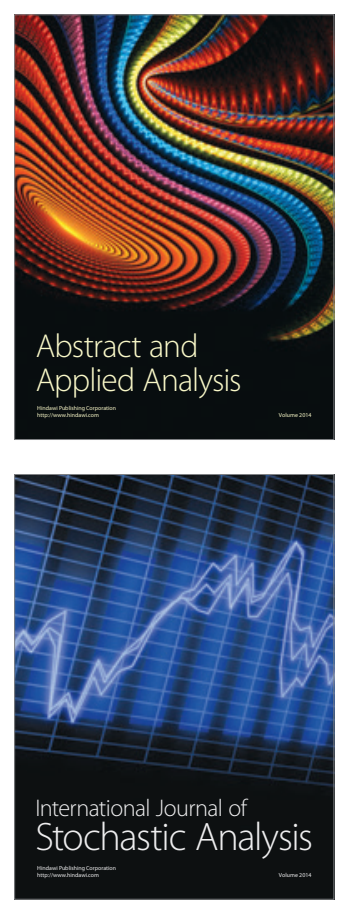

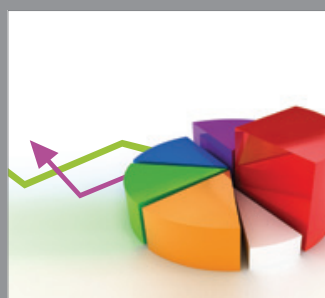

ournal of

Probability and Statistics

Promensencen
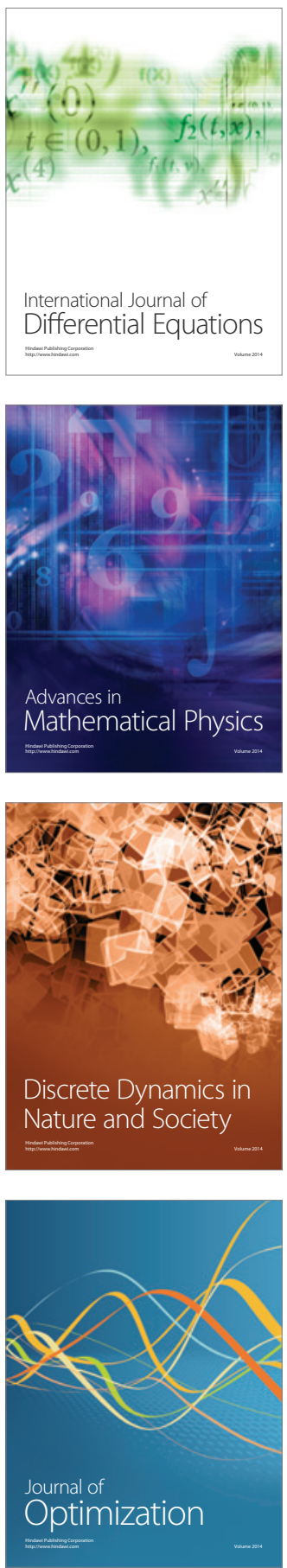